\title{
Auger Spectroscopy of Foil-Excited Beryllium Ions
}

\author{
R. Bruch, G. Paul, J. Andrä, and B. Fricke
}

\begin{abstract}
We have measured prompt and delayed emission spectra of electrons from foilexcited $\mathrm{Be}, \mathrm{Be}^{+}$, and $\mathrm{Be}^{2+}$ ions at $300 \mathrm{keV}$. On the basis of recently calculated eigenvalues we identified two lines in the prompt $\mathrm{Be}^{+}$spectrum as transitions from $2 s^{2} 2 p$ and $2 s 2 p^{2}$. The delayed Be spectrum indicates that transitions from highly excited quintet states occur. We propose radiationless deexcitation with one excited spectator electron not involved in the transition.
\end{abstract}

We have used the interaction of fast ionic beams $(300 \mathrm{keV})$ with thin solid carbon targets $\left(7 \mu \mathrm{g} / \mathrm{cm}^{2}\right)$ to populate highly excited ionic states in beryllium (Martinson, 1974), which subsequently decay either radiatively by electric dipole transitions or via autoionization to an adjacent electron continuum. The channels for autoionization of these states depend on the extent of configuration interaction with states with the same $L, S$, and $J$ values and parity. Auger transitions allowing the variation of $L, S$, or $J$ are only possible when the weaker spin-orbit $\left(H_{\mathrm{so}}\right)$, spin-other-orbit $\left(H_{\mathrm{soo}}\right)$, spin-spin $\left(H_{\mathrm{ss}}\right)$, and hyperfine coupling $\left(H_{\mathrm{hf}}\right)$ are involved (Das, 1973; Sevier, 1972).

One advantage of the beam-foil technique is the possibility for timeresolved observation of the ion beam, which permits one to separate the prompt electron emission $\left(\tau<10^{-10} \mathrm{sec}\right)$ from the delayed spectra $(\tau>$ $10^{-10} \mathrm{sec}$ ) characteristic of levels decaying by $H_{\mathrm{so}}, H_{\mathrm{soo}}, H_{\mathrm{ss}}$, and $H_{\mathrm{hf}}$. We have used a cylindrical electron spectrometer (Sellin, 1973) for accepting electrons emitted at $\theta=42.3^{\circ} \pm 1^{\circ}\left(2 \Delta \theta=2^{\circ}\right.$; beam diameter $\left.\rho=1.5 \mathrm{~mm}\right)$ from

R. Bruch, G. PAul, and J. ANDrä Boltzmannstrasse 20, Germany. Heinrich-Plett-Strasse 40, Germany.
- Institut für Atom- und Festkörperphysik A, l Berlin 33, B. FRICKE - Gesamthochschule Kassel, 35 Kassel, 
a small beam volume on the downstream side of a thin carbon foil. This foil could be moved relative to the viewing region of the electron spectrometer to detect both the prompt and delayed spectra. The electron energies measured in the laboratory system are transformed to center-of-mass energies by standard calculations (Risley, 1974). The spread in beam energy and angular spread of the beam after foil excitation, together with the finite acceptance angle of the spectrometer, produces an effective energy resolution of $1.7 \%$ in the Be spectra, compared to the spectrometer resolution of $0.3 \%$ when applied to a stationary point source of electrons.

We obtained complex discrete electron spectra characteristic of multiplyexcited levels in various charge states, superimposed on a continuum electron background produced by direct collision processes. In spite of this complexity, with the help of theoretical estimates of energy eigenvalues, we were able to interpret many of the observed structures (Bruch et al., $1975 a, b)$. We note that a single level can often decay to several different final levels, giving rise to several spectral features of different intensities. On the basis of the electron configurations, we are able to compare the most probable decays with the observed lines. We have also measured decay curves for the spectrum near $102.4 \mathrm{eV}$ and can resolve it into two decays, one of

BALASHOV et al.

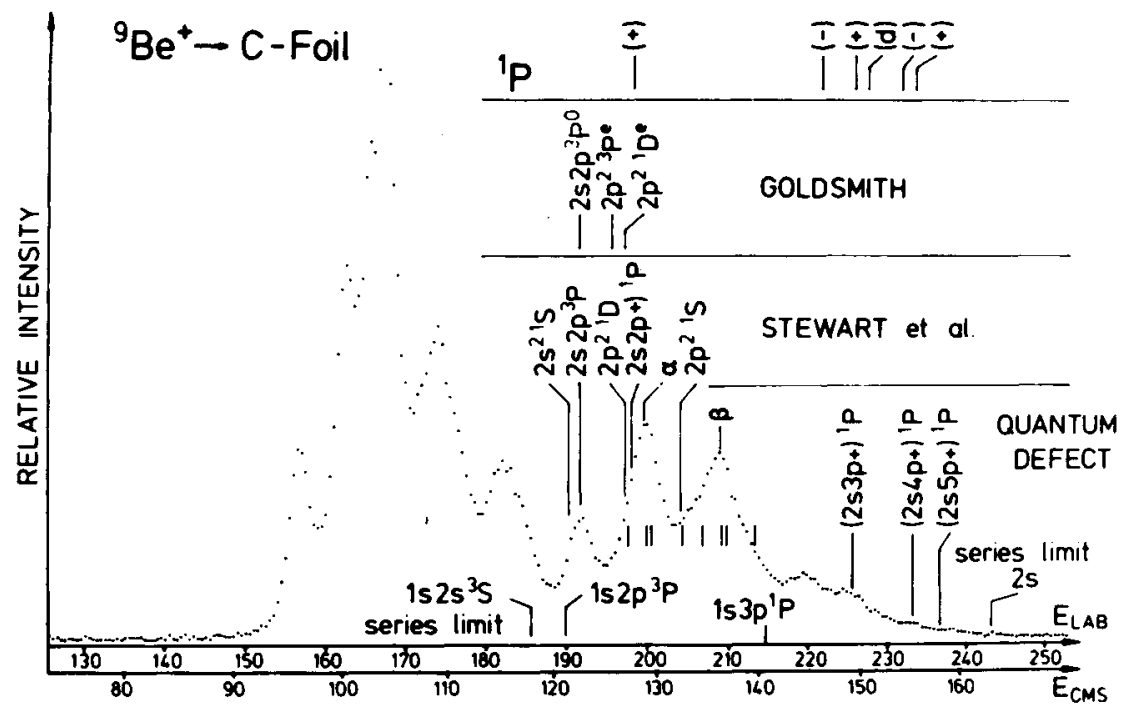

Figure 1. Electron spectrum of prompt autoionizing foil-excited $\mathrm{Be}, \mathrm{Be}^{+}$, and $\mathrm{Be}^{2+}$ states. (An absolute energy calibration $\pm 0.2 \mathrm{eV}$ in the cms system was achieved using the delayed $1 s 2 s 2 p\left({ }^{4} P_{5 / 2}\right) \rightarrow 1 s^{2} \varepsilon f\left({ }^{2} F_{5 / 2}\right)$ transition: $1 s 2 s 2 p\left({ }^{4} P\right)={ }^{4} P^{0}(1)$; see Figure 2.$)$ 
$3.1 \pm 0.4 \mathrm{nsec}$ lifetime and the other of $132 \pm 50 \mathrm{nsec}$. The shorter decay is mainly due to the optical dipole transition $1 s 2 p^{2}\left({ }^{4} P^{\mathrm{e}}\right) \rightarrow 1 s 2 s 2 p\left({ }^{4} P^{0}\right)$ measured by Hontzeas et al. (1973).

A prompt and a delayed spectrum is shown in Figures 1 and 2, respectively. The high-energy region of the prompt spectrum $\left(E_{\mathrm{cms}} \geqslant 120 \mathrm{eV}\right)$ is attributed to Be II and Be III transitions. This part of the spectrum is compared with: (a) transitions $2 \operatorname{lnl} l^{\prime}(n \geqslant 2) \rightarrow 1 s \varepsilon l^{\prime \prime}$, calculated from theoretical two-electron autoionization energies (Balashov et al., 1970; Chan and Stewart, 1967; Perrott and Stewart, 1968); (b) two-electron energy values deduced from optical dipole transitions, as observed in the far-ultraviolet Be III spectrum, produced by a vacuum spark (Goldsmith, 1969): and (c) transition energies of the Rydberg series $2 \operatorname{sn} p(n \geqslant 3) \rightarrow 1$ sep converging to the series limit $2 s \infty p \rightarrow 1 s \varepsilon p$, calculated with zero quantum defect for $3 p, 4 p$, and $5 p$ electron orbitals.

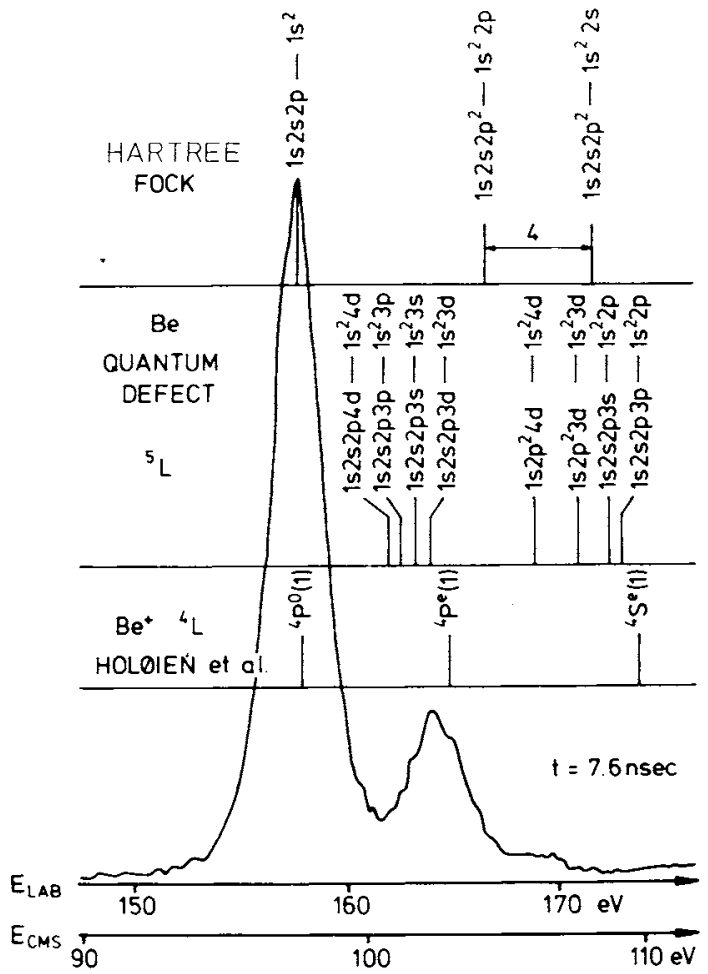

Figure 2. Delayed electron spectrum from foil-excited Be ions excited by impact on carbon foils. 
For Be II, series limits of the $\left(1 s n \ln ^{\prime} l^{\prime} \rightarrow 1 s^{2} \varepsilon l^{\prime \prime}\right)$ transitions are given in Figure 1 at $E_{\mathrm{cms}}=118.6 \mathrm{eV}\left[1 \mathrm{~s} 2 \mathrm{~s}\left({ }^{3} S\right)\right], E_{\mathrm{cms}}=121.9 \mathrm{eV}\left[1 \mathrm{~s} 2 p\left({ }^{3} P\right)\right]$, and $E_{\mathrm{cms}}=140.4 \mathrm{eV}\left[1 \mathrm{~s} 3 p\left({ }^{1} P\right)\right]$. In the energy region of interest, only the dominant lines of the $1 s n \ln ^{\prime} l^{\prime} \rightarrow 1 s^{2} \varepsilon l^{\prime \prime}$ series with $n$ and $n^{\prime} \geqslant 3$ should appear. No theoretical calculations are available yet for these series. However, in analogy to recent measurements of the $\left(3 / 3 l^{\prime}\right) \mathrm{H}^{-}$(Risley, 1974) and $\mathrm{Li}^{+}$(Bruch et al., 1975c) decays, one can assume that Be II transitions populating the $1 s 2 s$ and $1 s 2 p$ final ionic states should be much stronger than those decaying to the $1 s^{2}$ ground state. Due to this branching only small contributions from $1 \operatorname{sn} l n^{\prime} l^{\prime}\left(n, n^{\prime} \geqslant 3\right) \rightarrow 1 s^{2} \varepsilon l^{\prime \prime}$ transitions are expected in the energy range above $E_{\mathrm{cms}}=120 \mathrm{eV}$. The prominent peaks $\alpha$ and $\beta$ in the spectrum must therefore be assigned differently. They coincide with transition energies calculated recently (Lipsky and Ahmed, 1974) for the decay of $2 s^{2} 2 p\left({ }^{2} P^{0}\right)$ and $2 s 2 p^{2}\left({ }^{2} S^{\mathrm{e}}\right)$ states to $1 s 2 s\left({ }^{1,3} S\right)$ and $1 s 2 p\left({ }^{1,3} P\right)$ final ionic states, respectively. To our knowledge, no such promptly decaying three-electron autoionizing levels of positive ions have been observed previously (for the decay of negative ions see review article of Schulz, 1973). For the energy region $E_{\mathrm{cms}} \leqslant 120 \mathrm{eV}$, no complete comparison with theoretical calculations is yet possible. From first estimates, however, it is expected that the prominent features stem from three- and four-electron configurations (Bruch et al., 1975b).

Figure 2 shows part of the delayed Be spectrum 7.4 nsec after foil excitation and some theoretical energies estimated for highly excited threeand four-electron states. The $\mathrm{Be}^{+}$three-electron transitions among quartet states are assigned following the notion of Holøien and Geltman (1967). The ${ }^{4} P^{0}(1),{ }^{4} P^{\mathrm{c}}(1)$, and ${ }^{4} S^{\mathrm{e}}(1)$ states decay to the $\mathrm{Be}^{2+}$ state $1 s^{2}$. We believe that the discrepancy between the calculated position for $1 s 2 p^{2}\left({ }^{4} P^{\mathrm{e}}\right)={ }^{4} P^{\mathrm{e}}(1)$ $\left(E_{\mathrm{cms}}=103.1 \mathrm{eV}\right)$ and the center of the experimental peak $\left(E_{\mathrm{cms}}=102.4 \mathrm{eV}\right)$ is significant, in view of the reproducibility $\Delta E= \pm 0.2 \mathrm{eV}$ of the measurement. The theoretical estimate for the ${ }^{4} P^{\mathrm{e}}(1)-{ }^{4} P^{0}(1)$ energy separation (Holøien and Geltman, 1967) should be good to at least the same accuracy, and in fact fits data for quartet levels in $\mathrm{Be}^{+}$(Hontzeas et al., 1973) within experimental uncertainty. Be is of particular interest here, since it is the lowest $Z$ atom for which quintet states $\left({ }^{5} L\right)$ occur. From the metastable spectrum it is likely that three electron quartet states alone are insufficient to explain the data. Accordingly, we propose metastable quintet states as explanation, which decay via a channel with the orbit of a spectator electron $(3 s, 3 p, 3 d, 4 d)$ remaining unchanged. This decay mechanism is illustrated in Figure 3 for a typical Be I four-electron configuration $1 s 2 s 2 p 3 s$ decaying to four possible final ionic states $1 s^{2} 2 s\left({ }^{2} S\right), 1 s^{2} 2 p\left({ }^{2} P\right.$ ) (Junker and Bardsley, 1973), $1 s 2 s^{2}\left({ }^{2} S\right)$, and $1 s^{2} 3 s\left({ }^{2} S\right)$. Since the $2 s-2 p$ correlation is supposedly stronger than the $2 s-3 s$ and $2 p-3 s$ one, the dominant decay channel leads 


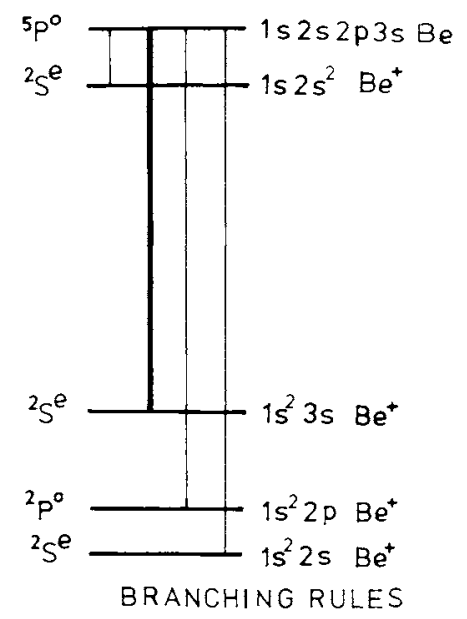

Figure 3. Decay scheme of the Be configuration $(1 s 2 s 2 p 3 s)$ with four open shells. (The scale is arbitrary; the $\mathrm{Be}^{+}$states shown are the final ionic states.)

probably to the $1 s^{2} 3 s\left({ }^{2} S\right)$ final state with the $3 s$ spectator electron unchanged (Krause et al., 1971).

The transition energies have been estimated using the quantum defect method (Rudd and Macek, 1974), with $\delta=0$ for $3 d$ and $4 d$ and $\delta \simeq 0.5$ for $3 s$ and $3 p$ orbitals (see Figure 2). Furthermore, Hartree-Fock calculations neglecting configuration interaction have been performed for the $1 s 2 s 2 p^{2} \rightarrow$ $1 s^{2} 2 p$ and $1 s 2 s 2 p^{2} \rightarrow 1 s^{2} 2 s$ transitions, where the $1 s 2 s 2 p$ parent configuration has been used for energy calibration of the results.

All these delayed quintet transitions, in particular, the group between 100.5 and $103.0 \mathrm{eV}$, can very well contribute to the observed spectrum. However, more accurate theoretical values for the transition energies, transition probabilities, and branching ratios of such four-electron transitions are needed for a clear-cut interpretation of the spectrum. Together with more advanced experimental techniques, such as ion-electron coincidence measurements for projecting out a definite final charge state, accurate calculations could help to classify a lot of yet unidentified lines.

\section{Acknowledgments.}

We are indebted to Professor J. Macek for many stimulating discussions. The authors wish to thank M. Ahmed and L. Lipsky for communication 
of their unpublished data. This work was supported by the Sonderforschungsbereich 161 der Deutschen Forschungsgemeinschaft.

\section{References}

Balashov, V. V., Grishanova, S. J., Kruglowa, H. M., and Senashenko, X. (1970). Opt. Spectr., 28. 466.

Bruch, R., Paul, G., and Andrä, J. (1975a). J. Phys. B., 8, L253.

Bruch, R., Paul, G., Andrä, J., and Fricke, B. (1975b). Phys. Lett., 53A, 293.

Bruch, R., Paul, G., Andrä, J., and Lipsky, L. (1975c). Phys. Rev., A12, 1808.

Chan, Y. M. C., and Stewart, A. L. (1967). Proc. Phys. Soc., 90, 619.

Das, T. P. (1973). Relativistic Quantum Mechanics of Electrons, 153 and 190.

Goldsmith, S. (1969). J. Phys. B., 2. 1075.

Holøien, E., and Geltman, S. (1967). Phys. Rev., 153, 81.

Hontzeas, S., Martinson. I., Enman, P., and Buchta, R. (1973). Nucl. Instr. Meth., $110,51$.

Junker, B. R., and Bardsley, J. N. (1973). Phys. Rev., A8, 1345.

Krause, M. O., Carlson. T. A., and Moddeman, W. E. (1971). J. Phys. (Paris) Colloq., C.4. 139.

Lipsky, L., and Ahmed, M. (1974). Private communication.

Martinson. I. (1974). Physica Scripta, 9. 281.

Perrott, R. H., and Stewart, A. L. (1968a). J. Phys. B., Ser. 2, 1, 381.

Perrott, R. H., and Stewart, A. L. (1968b), J. Phys. B., Ser. 2, 1, 1226.

Risley, J. S., Edwards, A. K., and Geballe, R. (1974). Phys. Rev., A9, 115.

Rudd, M. E., and Macek, J. H. (1974). Case Studies in Atomic Physics, III, 49.

Schulz, G. J. (1973). Rev. Mod. Phys., 45, 403.

Sellin, I. A. (1973). Nucl. Instr. Meth., 110, 477, and other references quoted there.

Sevier, K. D. (1972). Low' Energy Electron Spectrometry. 145. 